\title{
Hidatidosis cardiaca: caso clínico
}

\author{
CHRISTIAN ESPINOZA S. ${ }^{1}$, PEDRO PALMA V. ${ }^{2}$, \\ DIEGO SOTO V., ${ }^{3, \mathrm{a}}$, VANIA ROZAS A., ${ }^{3, \mathrm{a}}$, \\ ALFREDO RAMÍREZ N. ${ }^{4}$, MIGUEL GONZÁLEZ T. ${ }^{4}$
}

\section{Cardiac hydatidosis. Report of one case}

Hydatidosis is an endemic zoonosis in Chile. We report a 48-year-old former slaughterman, with a previous history of pulmonary hydatidosis, who presented a stroke without associated cardiovascular symptoms. An echocardiogram revealed a tumor mass with cystic features in the left ventricle. The patient was operated and the cyst was successfully excised. During the follow up, the patient remains asymptomatic.

(Rev Med Chile 2012; 140: 1449-1452).

Key words: Cysts; Echinococcosis; Thoracic surgery.
'Servicio Cardiovascular, Instituto Nacional del Tórax. Santiago, Chile. ${ }^{2}$ CESFAM Clotario Blest. Santiago, Chile.

${ }^{3}$ Universidad de Santiago de Chile. Santiago, Chile.

${ }^{4}$ Hospital Regional de Rancagua. Rancagua, Chile. anterno de Medicina.

Recibido el 11 de febrero de 2012, aceptado el 29 de junio de 2012

Correspondencia a: Dr. Christian Espinoza Silva Cirugía Cardiovascular, Instituto Nacional del Tórax José Manuel Infante 717 Providencia, Santiago. Celular: 09-4792277 E-mail:cardiocirugia@ gmail.com:
L a hidatidosis es la infección de animales herbívoros o del hombre con la larva del céstodo Echinococcus granulosus, parásito cuyo huésped definitivo es el perro y otros cánidos, que afecta a las regiones ganaderas.

En América Latina afecta mayormente a Argentina, Uruguay, Brasil y Chile ${ }^{1}$. En nuestro país, se producen anualmente alrededor de 800 casos nuevos de hidatidosis con una mortalidad que llega al 6-7\%. La enfermedad afecta a cualquier edad, siendo más frecuente entre los 10 y 60 años $^{2}$.

El compromiso cardiaco es raro, representando $0,5-3 \%$ de los quistes hidatídicos en humanos ${ }^{3}$. Las áreas de compromiso cardiaco incluyen el ventrículo izquierdo (60\%), ventrículo derecho (10\%), pericardio $(7 \%)$, arteria pulmonar $(6 \%)$, e inusualmente el tabique interventricular $(4 \%)^{4}$.

Las manifestaciones clínicas son extremadamente variables, relacionándose directamente con la localización y tamaño del quiste. El dolor precordial es el síntoma más común, aunque $90 \%$ de los pacientes son asintomáticos ${ }^{5}$. Otras manifestaciones posibles pueden ser pericarditis, taponamiento cardiaco, embolias sistémicas y shock anafiláctico severo ${ }^{6}$. Por este motivo el diagnóstico exige un alto grado de sospecha, ya que la sintomatología suele deberse a complicaciones, muchas veces fatales ${ }^{6}$.

Presentamos el caso de un paciente con antecedentes de hidatidosis pulmonar antigua, quien quince años después, presentó un accidente vascular encefálico sin sintomatología cardiovascular asociada, encontrándose un quiste cardiaco en una ecocardiografía. Resaltamos la importancia del diagnóstico y tratamiento precoz y la utilidad de las técnicas de diagnóstico por imagen, por el alto riesgo que conlleva esta condición.

\section{Caso clínico}

Varón de 48 años, oriundo de Marchigüe, VI Región de Chile, quien durante su vida laboral trabajó como faenador de corderos. Tenía antecedentes de hipertensión arterial, dislipidemia y un quiste hidatídico pulmonar operado en 1996. Quince años después, presentó un accidente vascular encefálico, sin referir otro tipo de sintomatología cardiovascular. Dentro del estudio etiológico se realizó un ecocardiograma y una tomografía computarizada de tórax (TAC) donde se evidenció una masa quística de $4 \times 3 \mathrm{~cm}$ en el ventrículo izquierdo, sin signos de ruptura. Se realizó además una ecotomografía abdominal que descartó la presencia de quistes hepáticos. Tampoco tenía antecedentes o síntomas previos sugerentes de anafilaxia.

Posteriormente, evolucionó con dolor precordial opresivo sin irradiación, de intensidad EVA 6/10. Evaluado por cardiología en su hospital base se indicó anticoagulación oral siendo derivado al Instituto Nacional del Tórax con la sospecha de hidatidosis cardiaca.

El paciente ingresó en buenas condiciones generales, afebril y sin alteraciones hemodinámi- 
cas, con presión arterial de 130/85 mmHg y 90 latidos por min. Al examen físico no presentaba ingurgitación yugular, roces ni soplos cardiacos (que fueron exhaustivamente buscados) ni tampoco signos de insuficiencia cardiaca. El examen pulmonar fue normal.

El hemograma reveló una eosinofilia de $21 \%$, sin leucocitosis. El perfil bioquímico fue normal. La determinación de anticuerpos IgG anti-Echinococcus granulosus no fue realizada.

La radiografía de tórax era normal y el electrocardiograma presentaba ritmo sinusal, ondas T negativas en DII y alteraciones inespecíficas en la repolarización.

La ecocardiografía realizada en nuestro servicio evidenció una imagen poliquística, ovalada y adherida al macizo de músculos papilares anterolaterales, de contornos bien delimitados en

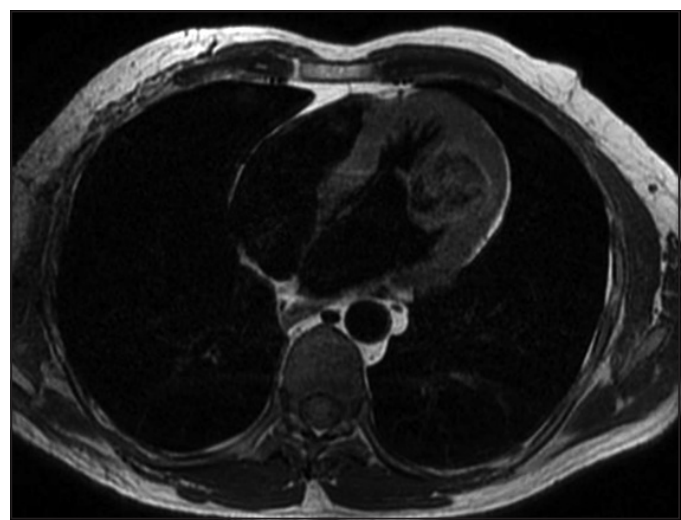

Figura 1. Resonancia nuclear magnética donde se aprecia una lesión ovoidea multi-vesiculada, adherida a la pared lateral del ventrículo izquierdo.

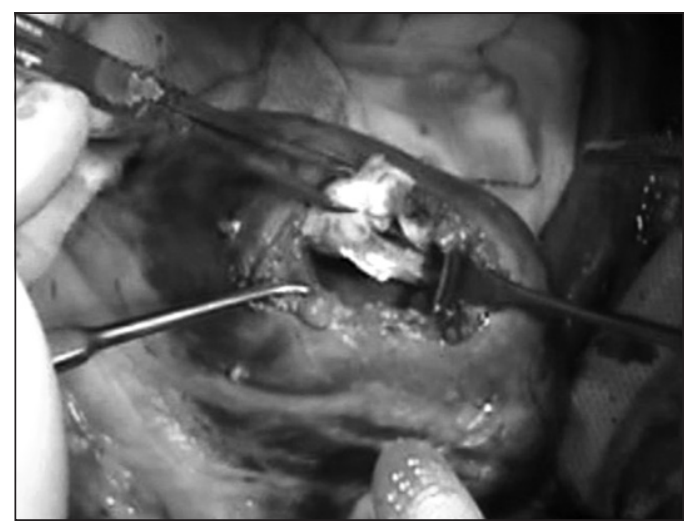

Figura 2. Resección de Quiste hidatídico y periquística a través ventriculotomía izquierda. pared libre del ventrículo izquierdo que medía $4,5 \mathrm{x}$ $3,2 \times 2,7 \mathrm{~cm}$, sin derrame pericárdico. Estos hallazgos fueron confirmados por resonancia magnética (RNM) (Figura 1). El estudio preoperatorio fue completado con una coronariografía, que fue normal.

La cirugía se realizó bajo esternotomía media y circulación extracorpórea, poniendo principal atención en la manipulación cardiaca para no lesionar el quiste. Una vez puesto el clamp aórtico, se disecó la superficie del quiste, se aisló el campo con compresas embebidas en cloruro de sodio al $10 \%$ y se puncionó el quiste vaciándolo parcialmente e inyectando solución salina hipertónica al 30\%. Posteriormente, se abrió el quiste hacia la cara anterior del ventrículo izquierdo y se vació, retirando las membranas y vesículas hijas. Se completó el aseo de la cavidad con sodio hipertónico y posteriormente se realizó quistectomía parcial y cierre por capitonaje del remanente (Figura 2). El paciente no presentó complicaciones intraoperatorias y la evolución posterior fue favorable. En control ambulatorio post-quirúrgico, el paciente se ha mantenido asintomático, sin evidencias de complicaciones o recidivas y se controlará semestralmente con ecocardiografía y serología para E. granulosus.

\section{Discusión}

La hidatidosis es una infección parasitaria causada por la ingestión de huevos de Echinococcus granulosus, que desarrollan posteriormente larvas enquistadas dentro del humano. La incidencia mundial se estima en 0,4 casos $/ 100.000$ personas $^{7}$. En Chile, la tasa de incidencia se ha mantenido estable (2-2,5 casos/100.000 habitantes), siendo las regiones más afectadas Aysén, Magallanes y Coquimbo ${ }^{8}$. Si bien nuestro paciente no tiene el factor de riesgo geográfico ${ }^{8}$, si tiene un claro antecedente de contacto habitual con ganado y de haber desarrollado hidatidosis pulmonar, determinando un mayor riesgo de reinfección.

Los órganos mayormente afectados son el hígado (50-79\%), pulmón (20\%) y en $10 \%$ de los pacientes ${ }^{9}$ se comprometen otros órganos. Existe daño multivisceral en 55-85\% de los casos, donde se compromete hígado, pulmón o ambos además de otros órganos ${ }^{10}$.

El E. granulosus puede llegar al corazón a través de la circulación coronaria, de un foramen oval permeable o de la circulación pulmonar. Cualquier parte del corazón puede ser afectada, siendo la localización más frecuente la pared libre del ventrículo izquierdo (50-77\%). En 50\% de los casos hay compromiso pericárdico ${ }^{11}$. Las contracciones miocárdicas pue- 
den afectar el proceso de crecimiento del quiste, induciendo un desarrollo multivesicular y aumentando la tensión sobre los quistes, facilitando su ruptura $^{12}$.

El 90\% de los pacientes son asintomáticos, con una clínica variable en relación con la región cardiaca afectada. Los quistes hidatídicos del ventrículo izquierdo son frecuentemente subepicárdicos e inusualmente se rompen, en cambio, los quistes ventriculares derechos generalmente son subendocárdicos siendo su ruptura más frecuente, generando una embolia pulmonar. Los quistes ubicados en el septum producen trastornos de la conducción y bloqueos atrioventriculares y la ubicación intracardiaca puede generar destrucción valvular ${ }^{13}$. En nuestro caso, el paciente se encontraba asintomático a pesar de la localización y tamaño del quiste $(5 \mathrm{~cm})$, lo que coincide con la literatura, donde se reportan quistes asintomáticos de hasta $7 \mathrm{~cm}$ de diámetro cuando el crecimiento es lento ${ }^{9,14}$.

Los métodos de detección de IgE específica, como el enzimoinmunoensayo (ELISA), tienen una especificidad que varía entre $77 \%{ }^{15}$ y $100 \%{ }^{16}$ en pacientes con hidatidosis. Sin embargo, la negatividad de las pruebas inmunológicas no descartan la equinococosis ya que algunos portadores de quistes no desarrollan anticuerpos detectables y su titulación varía según la localización anatómica (baja reactividad de quistes pulmonares, cerebrales y esplénicos) ${ }^{17}$. Por otra parte, los antígenos circulantes se detectan sólo en la mitad de los pacientes.

La combinación de imagen y serología usualmente provee un diagnóstico certero, aunque puede no tener buena sensibilidad ${ }^{18}$. La radiografía de tórax puede mostrar quistes torácicos, silueta cardiaca alterada o calcificaciones. La ecocardiografía, que muestra el quiste y sus características estructurales, juega un rol central en el diagnósti$\mathrm{CO}^{19}$ por su bajo costo y disponibilidad. Además se puede complementar con TAC o RNM.

Si bien no hay consenso, el tratamiento debiera ser una combinación de cirugía asociada a terapia antiparasitaria, ya que los resultados son mejores que el tratamiento médico exclusivo.

El tratamiento quirúrgico requiere bypass cardiopulmonar, hipotermia moderada y una técnica depurada, constituyendo una indicación de mediana urgencia, como fue nuestro caso. Las maniobras deben ser cuidadosas, evitando la ruptura del quiste y el vaciamiento del contenido a la circulación, la que puede tener hasta $75 \%$ de mortalidad secundario a hipersensibilidad y shock anafiláctico ${ }^{20} \mathrm{o}$ complicaciones embólicas ${ }^{21}$. Para evitar comprimir el ventrículo, éste se sostuvo con un sistema de aspiración continua "Starfish ${ }^{\infty}$ con el cual pudimos resecar completamente los elementos potencialmente infectantes. Durante el intraoperatorio y postoperatorio no se evidenció urticaria, angioedema o alteración hemodinámica que denotasen reacción anafiláctica.

Los protoescolicidas intraoperatorios son una técnica efectiva para eliminar el parásito. Los agentes más utilizados son las soluciones salinas hipertónicas (por su acceso económico y seguridad para el paciente $)^{22}$ por lo que decidimos utilizarla en nuestro caso. Sin embargo, nuevos agentes están siendo estudiados, como el gluconato de clorhexidina, el cual tendría mejores resultados en relación a la tasa de actividad protoescolicida ${ }^{23,24}$.

Con respecto a los antiparasitarios, optamos por una cobertura pre y postoperatoria con albendazol. Algunos grupos administran benzimidazólicos en el preoperatorio ${ }^{25}$ y otros los utilizan a partir del intraoperatorio o post-operatorio ${ }^{26}$, para disminuir el riesgo de diseminación y recurrencias $^{27}$.

El tratamiento preoperatorio con benzimidazolicos ayudaría a disminuir la viabilidad del quiste, lo que se correlaciona con una disminución de los títulos de IgE totales, IgE específicos y hemaglutinación indirecta ${ }^{17}$, que corresponden a signos indirectos de viabilidad y actividad del quiste. En nuestra opinión, el tratamiento de la hidatidosis es por excelencia quirúrgico, aunque existen situaciones donde el tratamiento antiparasitario es clave (quistes inextirpables o coadyuvante quirúrgico en quistes rotos pre y post cirugía ${ }^{28}$. El albendazol es el antiparasitario más utilizado, ya que sus características farmacocinéticas lo hacen superior con respecto a otros benzimidazólicos ${ }^{29}$, $\mathrm{y}$ es particularmente efectivo en quistes menores a $5 \mathrm{~cm}^{30}$.

No obstante la prolijidad del tratamiento, el control postoperatorio debe ser con imágenes repetidas hasta por 3 años, ya que la recurrencia puede ser tardía.

\section{Referencias}

1. Czischke C, Lorca M, Pinto P. Hidatidosis pulmonar. En: Rodríguez J, Undurraga A, Editores, Enfermedades Respiratorias. Santiago, Chile. Editorial Mediterráneo Ltda.; 2004 p. 715-29.

2. Sapunar J. Hidatidosis. En: Atías A, Editor, Parasitología 
Médica. Santiago, Chile. Editorial Mediterráneo Ltda.; 1998 p. 338-54.

3. Aydogdu T, Sahin N, Ulusan V, Gürpinar F, Türkay C, Bayezid Ö. Right atrial hydatid cyst associated with multiple organ involvement: Case report. J Thorac Cardiovasc Surg 2001; 121 (5): 1009-11.

4. Dursun M, Terzibasioglu E, Yilmaz R, Cekrezi B, Olgar S, Nisli K, et al. Cardiac Hydatid Disease: CT and MRI Findings. Am J Roentgenol 2008; 190 (1): 226-32.

5. Lagera S, García-Enguita M, Martínez-Gutiérrez F, Ortega J, Gutiérrez-Rodríguez A, Urieta A. A case of cardiac hidatidosis. Br J Anaesth 1997; 79 (5): 671-3.

6. Kadaras F, Kardara D, Tselikos D, Tsoukas A, Exadactylos N, Anagnostopolou M, et al. Fifteen year surveillance of echinococcal heart disease from a referral hospital in Greece. Eur Heart J 1996; 17 (8): 1265-70.

7. Tsigkas G, Chouchoulis K, Apostolakis E, Kalogeropoulou C, Koutsogiannis N, Koumoundourou D, et al. Heart echinococcus cyst as an incidental finding: early detection might be life-saving. J Cardiothorac Surg 2010; 5 (1): 124.

8. Cortés A, Valle B. Hidatidosis humana: Generalidades y situación epidemiológica en Chile según egresos hospitalarios y notifiación obligatoria entre los años 2001 y 2005. Rev Chil Infect 2010; 27 (4): 329-35.

9. Solano M, Urbieta M, Álvarez M, González C, Llorente B. Taponamiento cardiaco por quiste hidatídico pericárdico. An Med Interna (Madrid) 2005; 22 (7): 326-8.

10. Miralles A, Brancamonte L, Pavie A, Bors V, Rabago G, Gandjbakhch I, et al. Cardiac Echinoccocosis. Surgical treatment and results. J Thorac Cardiovasc Surg 1994; 107 (1): 184-90.

11. Ruiz Nodar J, Iturralde E, Aguilar R, Caniego J, Martínez de La Concha L, Martínez Elbal L. Rotura de un quiste hidatídico cardiaco situado en ventrículo derecho. Rev Esp Cardiol 1995; 48 (8): 563-5.

12. Gómez R, Otero E, Chorro F, Muñoz J, Losada A, López V. Quistes cardiacos. Un caso de hidatidosis cardiaca aislada. Rev Esp Cardiol 1999; 52 (5): 355-358.

13. Kaplan M, Demirtas M, Cimen S, Ozler A. Cardiac Hydatid Cysts with Intracavitary Expansion. Ann Thoracic Surg 2001; 71 (5): 1587-90.

14. Frider B, Larrieu E, Odrizola M. Long term outcome of asymptomatic liver hydatidosis. J Hepatol 1999; 30: 228-31.

15. Afferni C, Pini C, Misiti-Dorello P, Bernardini L, Conchedda M, Vicari G. Detection of specific IgE antibodies in sera from patients with hydatidosis. Clin Exp Immunol 1984; 55: 587-92.

16. Vildózola H, Espinoza I, Roldan W. Estandarización de una prueba de ELISA para detectar anticuerpos IgE en pacientes con equinococosis quística y su utilidad en el diagnóstico y seguimiento de pacientes tratados con albendazol: reporte preliminar. An Fac Med 2012; 73 (1): 35-41.

17. Matheu V, Gracia M, Rodríguez V, Olalde S, Baeza M. Shock anafiláctico secundario a rotura espontánea de quiste hidatídico esplénico. Rev Esp Alergol Inmunol Clín 1997; 12 (4): 242-7.

18. Barbieri M, Severi M, Pirez M, Battistoni J, Nieto A. Use of specific antibody and circulating antigen serum levels in the hydatid inmunodiagnosis of asymptomatic population. Int J Parasitol 1994; 24 (7): 937-42.

19. Ben-Hamda K, Maatouk F, Ben Farhat M, Betbout F, Gammra H, Addad F, et al. Eighteen years experience with echinoccocosus of the heart: clinical and echocardiografic features in 14 pacientes. Int J Cardiol 2003; 91 (2-3): 145-51.

20. Bertó J, Martínez F, Ojeda J. Shock anafiláctico como manifestación de hidatidosis hepática. Rev Clin Esp 1991; 188 (1): 60.

21. Unlu Y, Ceviz M, Karaoglanuglu N, Becit N, Kocak H. Arterial embolism caused by a ruptured hdatyd cyst in the heart: report a case. Surg Today 2002; 32 (11): 989-99.

22. Neira P, Lorca M. Echinococcus granulosus: evaluation of protoscolicidal activity of several substances. Rev Patol Tropical 2001; 30 (1): 93-9.

23. Puryan K, Karadayi K, Topcu O, Canbay E, Sumer Z, Turan $\mathrm{M}$, et al. Chlorhexidine gluconate: an ideal scolicidal agent in the treatment of intraperitoneal hydatidosis? World J Surg 2005; 29 (2): 227-30.

24. Topcu O, Sumer Z, Tuncer E, Aydin C, Koyuncu A. Efficacy of Chlorhexidine gluconate during surgery for hydatid cyst. World J Surg 2009; 33 (6): 1274-80.

25. Tuncer E, Tas S, Mataraci I, Tuncer A, Donmez A, et al. Surgical treatment of cardiac hydatid disease in 13 patients. Tex Heart Inst J 2010; 37 (2): 189-93.

26. Kaplan M, Demirtas M, Cimen S, Ozler A. Cardiac Hydatid Cysts with intarcavitary expansion. Ann Thorac Surg 2001; 71 (5): 1587-90.

27. Moro P, Schantz P. Echinococcosis: a review. Int J Infect Dis 2009; 13 (2): 125-133.

28. Noemí I, Viovy A, Zamorano R, Blanco A, Revello D, Vojkovic M, et al. Hidatidosis en la infancia: Albendazol en su tratamiento médico y quirúrgico. Rev Chil Infect 2003; 20 (4): 229-34.

29. Atías A. El tratamiento médico de la hidatidosis. Parasitología al día. 1993; 17: 153-7.

30. Apt W, Pérez C, De Rycke P. Tratamiento no convencional de la hidatidosis humana. Rev Med Chile 1996; 124: 1385-9. 